sierend auf die krankhaft veränderten Schleimhäute zu wirken. Der Gang der Behandlung ist folgender: Zuerst werden die Borken durch $2-$ roproz. Salicylvaseline entfernt, darauf beginnt eine intensive Bepinselung der ganzen Schleimhaut mit Stieltupfer und Wattebausch mit 5proz. spirituöser Yatrenlösung, dann folgt Einblasung von Yatren purissimum. Diese letzteren beiden Prozeduren sollen täglich 2-3mal später I-2mal bis rmal am Tag ausgeführt werden. Bei erneuter geringer Borkenbildung kann mit Salicylvaseline wieder abgelöst werden. Die Resultate sind sowohl bei Ozaena auf hereditär luetischer Basis wie bei Ozaena ohne diese Basis gleich gute; schon nach $\mathrm{I}_{4}$ Tagen läßt der Geruch fast vollständig nach. Im allgemeinen waren bis jetzt alle 5 Patienten schon nach 4 Wochen ohne jegliche Beschwerden, selbst die Borkenbildung ist nur minimal. Es ist zu empfehlen, daß die Patienten, die nunmehr beruflich und gesellschaftlich ungehemmt sind und in der kurzen Zeit der Behandlung sichtlich aufleben, sich nach kurzen Pausen unter Kontrolle des Arztes selbst*) weiter behandeln, um Rezidive zu vermeiden.

\section{ÜBER DEN VITAMINGEHALT DES DIÄTETISCHEN NÄHRPRÄPARATES „HYGIAMA“.}

Von

Dr. Wilhelm Brauch, Basel.

Aus der Medizinischen Universităts-Poliklinik Basel.

Die Existenz der Vitamine, deren chemische Konstitution man bisher noch nicht hat ermitteln können, deren physikalische Eigenschaften wie Löslichkeit, Hitzewiderstandsfähigkeit usw. bekannt sind, gilt heute wohl allgemein als gesichert in der Wissenschaft.

Die unzähligen Vitaminarbeiten des letzten Jahrzehntes ergaben die große Bedeutung der Vitamine in der modernen Ernährungslehre. Die Beurteilung des physiologischen Wertes einer Nahrung, eines Nährmittels, muß deshalb heute von einem erweiterten Standpunkt aus wie früher erfolgen. Neben der Frage nach der chemischen Zusammensetzung und dem Energieinhalt ist die Frage nach dem Vitamingehalt gleichberechtigt, wenn nicht oft noch wichtiger.

Ein künstliches Nährmittel, das zugleich diätetisches Heilmittel sein will, muB also nicht nur in chemischer, physikalischer und physiologischer Hinsicht vollwertig sein, d. h. Eiweiß, Kohlenhydrate, Fett, Salze in für die Verdauung geeigneter Form und richtigem Mengenverhältnis enthalten, es muß auch, besonders wenn es zeitweise als alleiniges Nahrungsmittel gebraucht werden soll, die 3 Vitamingruppen A, B, C in genügender Menge enthalten.

Unter unseren europäischen Verhältnissen sind dank der verschiedenartigen Ernährungsmöglichkeiten besonders bei Erwachsenen Krankheiten durch Vitaminmangel wie Skorbut und Beriberi äußerst selten. Dagegen tritt nach der Anschauung vieler Autoren hauptsächlich infolge A-Vitaminmangel, besonders bei Kindern, Rachitis verhältnismäBig oft auf. Für die Beurteilung eines künstlichen Nährmittels ist deshalb die Frage nach dem AVitamingehalt besonders wichtig.

Wir haben für unsere Untersuchungen das altbekannte diätetische Nähr- und Heilpräparat "Hygiama" der Dr. Theinhardts Nährmittel-Gesellschaft A.-G., Stuttgart-Cannstatt deshalb gewählt, weil uns nicht nur die chemische Zusammensetzung und der Energieinhalt bekannt ist, sondern weil wir auch über die Herstellungsweise genauer orientiert sind.

Die Durchschnittsanalyse ergibt für Hygiama:

\begin{tabular}{|c|c|}
\hline ser & $4,75 \%$ \\
\hline Eiw & $21,22 \%$ \\
\hline Fett. . & $10,05 \%$ \\
\hline lösliche Kohlenhydrat & 49,10 \\
\hline unlösliche Kohlenhydrate & II, 33\% \\
\hline $\begin{array}{l}+ \text { Mineralstoffe } \\
+ \text { darin Phosphor }\end{array}$ & $\begin{array}{l}3,55 \% \\
. \quad 1,05 \%\end{array}$ \\
\hline
\end{tabular}

In chemischer und energetischer Hinsicht ist Hygiama als gutes und vollwertiges Nährmittel zu bezeichnen.

Die Prüfung auf A-Vitamin, das man auch als Wachstums- und Antirachitisvitamin bezeichnet hat, kann auf verschiedene Weise geschehen. Oft benützt man hierzu junge, $40-50 \mathrm{~g}$ schwere Ratten, die bei einer A-Vitaminfreien Nahrung das Wachstum sofort einstellen und erst auf Zusatz von A-vitaminhaltiger Nahrung weiterwachsen.

Wir haben zur Prüfung auf A-Vitamin zunächst 2 etwa 6 Wochen alte Hunde benützt, die, nach dem Vorgange von Mellanby (Journ. physiol. 52, 52. 1919), mit einer A-vitaminfreien basalen Nahrung gefüttert, innerhalb 4-6 Wochen an rachitischen Erscheinungen erkranken sollen.

*) Zu diesem Zweck wird in kurzer Zeit ein handliches Besteckchen mit allem Notwendigen für die Selbstbehandlung in den Handel gebracht werden.
Die basale Nahrung bestand täglich pro Hund ans etwa:

Magermilch . . . $350 \mathrm{ccm}$ Hefe . . . . 2-3g Weizenweißbrot ad 35 Apfelsinensalt . . 3-5 ccm libitum Leinöl . . Io ccm Salzgemisch . . . I-2 g

Magermilch, Weizenweißbrot und Leinöl sind bekanntlich sehr arm an A.-Vitamin und deshalb besonders zur experimentellen Erzeugung der Rachitis sehr geeignet. Durch den Hefezusatz sollte genügend B-Vitamin, durch den Apfelsinensaft genügend C-Vitamin zugeführt werden.

Die beiden Hunde waren Geschwister, leider aus einer Kreuzung stammend, Hund I ein Fox, Hund 2 eine kleine Bulldogge, so daß die Gewichte und Gewichtszunahmen nicht direkt vergleichbar sind. Hund I erhielt nur die obige basale, rachitiserzeugende Kost, Hund 2 erhieit zu dieser Kost von Beginn des Versuches an täglich eine Extrazulage von etwa Io-zo g Hygiama, das mit Wasser zu einem Brei aufgekocht, per os eingegeben wurde.

Schon mach 5 Wochen zeigte Hund I beginnende Rachitis, die nach 6 Wochen sehr deutlich als solche zu erkennen war. Besonders die Vorderbeine sind stark, typisch rachitisch gekrümmt, beim Befühlen der Knochen und Gelenke unter leichtem Druck zeigt das TierSchmerzen. Es kann sich bedeutend schlechter bewegen und gehen als Hund 2., der nach dieser Zeit keinerlei rachitische Merkmale aufweist. Die Beine sind vollständig gerade und normal, beim Befühlen und Druck auf die Knochenund Gelenke zeigt das Tier keine Schmerzempfindung, Hund 2 ist durch Hygiamazulage gesund geblieben.

Aus diesem Stand des Versuches kann man schon den Befund erheben, das Hygiama A-Vitamin in genügender, reichlicher Menge enthält, denn der geringe Zusatz von täglich $10-20 \mathrm{~g}$ zur basalen, rachitiserzeugenden Nahrung hat den Hund 2 vor einer Erkrankung bewahrt.

Es war nun interessant und sehr wichtig zu wissen, ob durch nachträglichen Hygiamazusatz zur Nahrung des erkrankten Hundes $I$ die rachitischen Erscheinungen zurückgehen und verschwinden. Von der 7 . Woche ab erhielt Hund I auch Hygiamazusatz zur basalen Nahrung, die nicht geändert wurde. Hund 2 wurde gleich wie bisher gefüttert.

In der 15. Versuchswoche, also 8 Wochen nach Beginn des Hygiamazusatzes, waren bei Hund $\mathbf{I}$ die rachitischen Erscheinungen: Krumme Beine, schmerzende, schwache Gelenke, mühsames Gehen weitgehend zurückgegangen. Das Tier hat sich gut weiterentwickelt und sehr stark zugenommen, es kann sich ebenso gut bewegen wic Hund 2, der auch in der $\mathbf{I}_{5}$. Versuchswoche noch vollständig normal ist und auch stark an Gewicht zugenommen hat. Röntgenaufnahmen des rechten Vorderbeines bei Hund I und 2 am Ende der 15. Woche zeigten keinen rachitischen Befund, Knochen und Gelenke waren bei beiden Hunden normal.

"Hygiama" als Zusatz zu einer A-vitaminarmen Nahrung ist also imstande, sowohl einer Rachitiserkrankung vorzubeugen, als auch eingetretene Rachitis weitgehend zu heilen.

Dieses Ergebnis gab Veranlassung, die Versuche in üblicher Weise mit weißen, jungen Ratten zu kontrollieren, sowohl mit Hygiama als auch mit dem verwandten "Infantina".

Die vitaminfreie Grundnahrung bestand aus: 18\% Casein, I $5 \%$ Schweinefett, $2 \%$ Cellulose, $6 \%$ Salzgemisch, $59 \%$ Stärke. Zur Sicherung der Vitaminfreiheit wurden das Casein und die Stärke nochmals mit Alkohol extrahiert und das Schweinefett während mehrerer Stunden unter Luftdurchleitung erhitzt. Junge, etwa $40-50$ g schwere weiße Ratten, damit ausschließlich gefüttert, zeigen keine Gewichtszunahme, sondern gehen nach io-3o Tagen meist ein. Bei einer Zulage von täglich nur $0,5 \mathrm{~g}$ Butterfett (A-Vitamin) und $0,5 \mathrm{~g}$ Hefe (B-Vitamin) zeigen die Tiere jedoch schönes Wachstum.

Zur Prüfung auf A-Vitamin wurden 2 Ratten angesetzt: Ratte $x$ erhielt zur Grundnahrung etwa 20\% Hygiama, Ratte 2 zur Grundnahrung etwa $20 \%$ Infantina, dazu beide Ratten täglich $0,5 \mathrm{~g}$ Hefe

Bei Vorhandensein von A-Vitamin in den beiden Präparaten mußten die beiden Ratten gutes Wachstum zeigen. Beifolgend die Gewichte der Ratten während des Versuches:

$$
\begin{aligned}
& \text { Bei Beginn des Versuches } 5 \mathrm{I} \mathrm{g} \quad 5^{6} \mathrm{~g} \\
& \text { Nach } 6 \text { Tagen.... } 66 \mathrm{~g} \quad 60 \mathrm{~g} \\
& \text { Iо ". . . } 66 \mathrm{~g} \quad 62 \mathrm{~g} \\
& 17 \quad, \quad . .278 \mathrm{~g} \quad 69 \mathrm{~g} \\
& 22, . .293 \mathrm{~g} \quad 85 \mathrm{~g} \\
& 28 \text { ". . . . I02 g } 94 \mathrm{~g} \\
& \text { 3I . . . . . } 107 \mathrm{~g} \text { roo g }
\end{aligned}
$$

Im Laufe eines Monates haben also beide Ratten ihr Gewicht verdoppelt, das rasche Wachstum der beiden Versuchstiere bestätigt also in bezug auf Hygiama das Ergebnis des Hundeversuches und stellt bei Infantina das Vorhandensein von reichlich A-Vitamin fest.

Zusammenfassung: Durch Fütterungsversuch am Hund wird festgestellt, daß das diätetische Nährmittelpräparat Hygiama reich A-Vitaminhaltig ist. Durch Fütterungsversuch an der Ratte wird dieses Ergebnis bestätigt und bei dem verwandten Präparat „Infantina" reichlicher A-Vitamingehalt neu festgestellt. 